\title{
Effects of Chemical Modification on Physical And Mechanical Properties of Rice Husk - Stripped Oil Palm Fruit Bunch Fiber Polypropylene Hybrid Composite
}

\author{
${ }^{1}$ M.A. Hassan, ${ }^{2}$ O.S. Onyekwere, ${ }^{3}$ A.M. Yami, ${ }^{4}$ A. Raji \\ ${ }^{1,2,3,4}$ Department of Mechanical Engineering, Modibbo Adama University of Technology, Yola
}

\begin{abstract}
Rice husk and stripped oil palm fruit bunch were used as reinforcing filler in the preparation of particle-reinforced hybrid composite with polypropylene as the matrix. To determine the effect of chemical modification of the lignocellulose materials on the mechanical properties of the hybrid thermoplastic polymer composites, the reinforcing fibers were divided into three parts the crude (untreated), mercerized and acetylated parts. Three levels of the filler loading $(20,30$ and $40 \mathrm{wt} \%)$ were used in the experimental design. The test samples were produced by compounding the fibers with polymer matrix at temperature range of $165^{\circ} \mathrm{C}$ to $175^{\circ} \mathrm{C}$ and subsequently molded at a pressure of 32MPa. The test samples were subjected to mechanical properties tests and the results analyzed using ANOVA at highly significant level of 0.01 and significant level of 0.05. The results show that mercerization caused significant increase in the tensile strength, impact strength and hardness values of the hybrid composite. The same treatment showed no significant effect on the values of compressive strength, tensile modulus, density and percentage water absorption. Acetylation tended to cause reduction in water absorption, improved impact and tensile strengths and hardness value while tensile modulus and compressive strength of the samples decreased due to acetylation. No significant effect on the density of the hybrid composites, consequent to acetylation, was observed from obtained results.
\end{abstract}

Keywords: Rice husk, stripped oil palm fruit bunch, chemical modification, properties

\section{Introduction}

Natural fibers are inherently incompatible with hydrophobic thermoplastics because they are hydrophilic in nature as they are derived from lignocelluloses. The major limitations of using natural fibers as reinforcement in thermoplastic matrixes include poor interfacial adhesion between polar-hydrophilic fiber and non polar-hydrophobic matrix, and difficulty in mixing due to poor wetting of the fiber with the matrix. According to [1], this in turn would lead to composites with weak interface. Bio-fibers interfacial adhesion with the matrix is enhanced by chemical modification. A strong interface can assure that the composite is able to bear load. The chemical modifications carried out in this work include; retting, scouring, mercerization and acetylation. Retting and scouring were carried out to purify the fibers. Scouring was mainly carried out to remove waxes and fats. Mercerization is an alkali treatment which imposes the fiber-matrix adhesion due to the removal of natural and artificial impurities. Alkali treatment also leads to fibrillation which causes the breaking down of the composite fibers into smaller fibers. Hence, the development of a rough surface topography offer better fiber matrix interface adhesion and an improvement in mechanical properties [2]. The main principle of acetylation is to react the hydroxyl groups $(\mathrm{OH})$ of the fiber with acetyl groups $\left(\mathrm{CH}_{3} \mathrm{CO}\right)$ thereby rendering the fiber surface more hydrophobic [3]. Acetylation has been shown to reduce swelling of wood in water and to enhance the interface in flax/polypropylene composite [4].

In this study, rice husk and stripped oil palm fruit bunch were subjected to two chemical modifications - mercerization and acetylation, after purification of the fiber. The properties of the composites produced with the chemically modified fibers were compared to the properties of the untreated (crude) fiber composites.

\subsection{Materials}

\section{Materials and Methods}

Commercial polypropylene (PP), supplied by HAN WHA L and C Corp. South Korea in the form of homopolymer pellets, was used as the polymer matrix. The density of the PP was $0.91 \mathrm{~g} / \mathrm{cm}^{3}$, melt flow index (MEI) $12 \mathrm{~g} / 10 \mathrm{~min}$ (Temperature $230^{\circ} \mathrm{C} / 2160 \mathrm{~g}$ ). Rice husk, which belongs to the specie oryza sativa, obtained from a local rice mill in Yola, Nigeria and stripped oil Palm fruit bunch (SOPFB) obtained from agro-farm in Aba, Nigeria were used as reinforcement filler in the composite. 
2.2. Methods

2.2.1. Fiber chemical modifications

The fibers were purified, in two stages, to remove artificial and natural impurities. In the first stage, $10 \mathrm{~g}$ of each type of fiber was placed in $100 \mathrm{ml}$ of $6 \% \mathrm{w} / \mathrm{v}$ ammonium oxalate solution in a beaker and its content was heated to boil for 45 minutes. Then the fibers were thoroughly rinsed under running water and dried. In the second stage of fiber purification, which was carried out mainly to remove waxes, fats and other mucilaginous substances, $10 \mathrm{~g}$ of each of the retted fibers were weighed into a beaker and $100 \mathrm{ml}$ of $2 \% \mathrm{w} / \mathrm{v} \mathrm{NaOH}$ added. The mixture was heated to boil for 45 minutes. It was then washed under running water and dried.

Part of the purified fibers was mercerized by immersion in $6 \% \mathrm{w} / \mathrm{v} \mathrm{NaOH}$ solution for 2 hours at room temperature [5]. After the alkaline treatment the fibers were thoroughly washed in water and dried at $80^{\circ} \mathrm{C}$ until a constant weight was reached.

Acetylating was carried out by first adding $5.5 \mathrm{~cm}^{3}$ of acetic acid, $2.5 \mathrm{~cm}^{3}$ of acetic anhydride and 0.65 $\mathrm{cm}^{3}$ of concentrated $\mathrm{H}_{2} \mathrm{SO}_{4}$ to each $1 \mathrm{~g}$ of fiber. The mixture was warmed gently to $45^{\circ} \mathrm{C}$ for one hour after which $0.65 \mathrm{~cm}^{3}$ of concentrated $\mathrm{H}_{2} \mathrm{SO}_{4}$ was again added and the whole mixture stirred using a magnetic stirrer and keeping the temperature at $45^{\circ} \mathrm{C}$ for another one hour. The fibers were then removed, immersed in water and allowed to stand for 48 hours after which they were dried.

\subsubsection{Composite formation}

Prior to composite formation, the treated and untreated rice husk $(\mathrm{RH})$ and stripped oil palm fruit bunch (SOPFB) fibers were each grinded and screened to $200 \mu \mathrm{m}$ mesh size. The sieved rice husk and SOPFB were then dried and kept in suitable containers. During composite formation, the required quantities of reinforcing fiber (10RH/10SOPFB, 10RH/20SOPFB, 20RH/10SOPFB or 20RH/20SOPFB) and polypropylene were transferred to an already pre-heated (to temperature of $75-80^{\circ} \mathrm{C}$ ) mixing container and continuously mixed with the aid of a stirrer while simultaneously increasing the temperature of the container until a homogeneous mixture is obtained at a temperature of $165-175^{\circ} \mathrm{C}$. The homogenous mixture was then poured into a mould and ho pressed at a pressure of $32 \mathrm{MPa}$ to obtain the samples. After cooling, the solidified samples were thereafter ejected and prepared for the required test.

\subsubsection{Testing}

\subsubsection{Physical tests}

The percentage water absorption was conducted according to ASTMD - 560. The samples were weighed and then immersed in water for 24 hours at room temperature. The percentage water absorption was calculated using (1):

$\%$ water absorption $=\frac{(W-D) \times 100}{D}$

where $\mathrm{D}=$ weight of dry sample, $\mathrm{W}=$ weight of soaked sample.

To calculate the density, the specimens were weighed in a weighing balance and their weights recorded. Their volumes were calculated from their dimensions. The density was calculated using (2):

$$
\text { Density }=\frac{\text { mass }}{\text { volume }}
$$

\subsubsection{Mechanical tests}

All mechanical tests were carried out at the temperature of $23 \pm 2{ }^{\circ} \mathrm{C}$ and relative humidity of $50 \pm$ $5 \%$. The tensile property measurements were carried out in a universal testing machine according to ASTM D638 at a crosshead speed of $5 \mathrm{~mm} / \mathrm{min}$. For compressive test, the specimens were cut to $25 \mathrm{~mm} \times 25 \mathrm{~mm} \times 12 \mathrm{~mm}$ and then ground with carbide sand paper in order to obtain smooth surface. The test was carried out in a universal testing machine in accord with ASTM D 695 - 96. Unnotched Izod impact strength test was conducted according to ASTM D - 256 at room temperature. The Brinnel hardness value was measured using Meyers apparatus for hardness test. Each value obtained represented the average of four samples.

\section{Results and Discussion}

Table 1 and Table 2 show the physical and mechanical properties of the developed composites respectively. Table 3 shows the statistical comparison of the physical and mechanical properties for crude, mercerized and acetylated fiber composites. Anova was used in the statistical analysis. The effect of chemical modification on properties of the composite was statistically analyzed at a significant level of 0.05 and highly significant level of 0.01 .

\subsection{Physical properties}

Table 1 shows percentage water absorption and density for crude, mercerized and acetylated fiber composites respectively. From the table, \% water absorption is seen to generally increase with increase in filler 
loading (from 1.39 to 10.92 for crude samples; from 4.38 to 10.81 and from 0.21 to 3.43 respectively for samples with crude, mercerized and acetylated fiber contents). For the same loading levels acetylation caused improvement in water absorption from 1.39 to 0.21 ; from 4.11 to 1.53 ; from 5.99 to 1.75 and from 10.92 to 3.43 respectively for 10RH/10SOPFB, 10RH/20SOPFB, 20RH/10SOPFB, and 20RH/20SOPFB samples. According to Table 3 , there is no significant difference between the percentage water absorption of crude and mercerized fiber composites. Generally, the percentage water absorption of acetylated fiber composites differ highly significantly $(\mathrm{P}<0.01)$ from both the crude and mercerized fiber composites. The mean difference shows that acetylating reduces the percentage water absorption of the fiber composites. Similar effect of acetylation on water absorption was reported by [6] for wood fiber and by [7] for Kenaf fiber composites. During the process of acetylation, the hydroxyl groups $(\mathrm{OH})$ of the fiber reacts with acetyl groups $\left(\mathrm{CH}_{3} \mathrm{CO}\right)$, thereby rendering the fiber surface more hydrophobic according to (3);

Fiber $-\mathrm{OH}+(\mathrm{CH} 3 \mathrm{CO}) 2 \mathrm{O} \rightarrow$ Fiber $-\mathrm{OOC} . \mathrm{CH} 3+\mathrm{CH} 3 \mathrm{COOH}$

Table 3 shows that, there is no significant difference in the densities for different chemical treatments $(\mathrm{P}>0.05)$ meaning that chemical modification had no significant effect on the density of the composites.

Table 1: Physical Properties of the Developed Hybrid Composite for Different Chemical Modifications

\begin{tabular}{|l|l|l|l|l|}
\hline PROPERTIES & COMPOSITIONS & CRUDE & MERCERIZED & ACETYLATED \\
\hline \multirow{5}{*}{ \% Water Absorption } & 10RH/10 SOPFB & 1.39 & 4.38 & 0.21 \\
& 10RH/20 SOPFB & 4.11 & 2.94 & 1.53 \\
& 20RH/10 SOPFB & 5.59 & 3.19 & 1.75 \\
& 20RH/20 SOPFB & 10.92 & 10.81 & 3.43 \\
\hline \multirow{5}{*}{ Density $\left(\mathbf{g} / \mathbf{c m}^{\mathbf{3}}\right)$} & $10 \mathrm{RH} / 10$ SOPFB & 0.75 & 0.72 & 0.80 \\
& $10 \mathrm{RH} / 20$ SOPFB & 0.73 & 0.73 & 0.72 \\
& 20RH/10 SOPFB & 0.72 & 0.73 & 0.67 \\
& 20RH/20 SOPFB & 0.68 & 0.67 & 0.66 \\
\hline
\end{tabular}

\subsection{Mechanical properties}

Table 2 shows the mechanical properties of the crude, mercerized and acetylated fiber composites. Tensile strength range from 22.54 to $27.27 \mathrm{MPa}$; from 27.14 to $29.67 \mathrm{MPa}$ and from 28.54 to $33.83 \mathrm{MPa}$ respectively for crude, mercerized and acetylated fiber-based composites. According to table 3 , the tensile strength of crude fiber composites differs highly significantly $(\mathrm{P}<0.01)$ from both mercerized and acetylated fiber-based composites. The tensile strength of mercerized fiber-based composite differ significantly $(\mathrm{P}<0.05)$ from acetylated fiber-based composite. Acetylated fiber composites showed higher tensile strength than mercerized fiber composites while mercerized fiber-based composites showed higher tensile strength than crude fiber composites. The poor tensile strength of composites with crude fiber contents is due to poor interfacial bonding between the hydrophilic filler and the hydrophobic matrix polymer which results in decreased tensile strength [8]. The improved tensile strength of the mercerized fiber-based composite over crude fiber-based composite could be as a result of increased surface roughness and decreased surface polarity which improve fiber-matrix interaction. During mercerization, $\mathrm{NaOH}$ cleans the fiber surface by removing impurities, waxes and part of lignin. Partial removal of lignin causes some debonding of the fibrils which leads to protrusion of some of them. Such protrusions, as observed by [5] and [9], produce mechanical bonding of the fibers and consequently improve fiber-matrix interaction. Improved tensile strength for acetylated fiber-based composites could be because of better interfacial bonding between acetylated fiber which has been rendered hydrophobic and the matrix polymer which by nature is hydrophobic.

As seen in Table 2, tensile modulus of the hybrid composites range from 259.60 to $398.12 \mathrm{MPa}$; from 308.99 to $373.15 \mathrm{MPa}$ and from 260.75 to $324.30 \mathrm{MPa}$ respectively for crude, mercerized and acetylated fiberbased composites. Statistical analysis in Table 3, shows that the tensile modulus of the acetylated fiber composite differs significantly from both crude and mercerized fiber composite $(\mathrm{P}<0.05)$. There is no significant difference between the tensile modulus of the crude and mercerized fiber composite $(\mathrm{P}>0.05)$. From the mean difference, the crude and mercerized fiber composite showed higher tensile modulus than the acetylated fiber composites.

Table 2: Mechanical Properties of the Developed Hybrid Composite for Different Chemical Modifications

\begin{tabular}{|l|l|l|l|l|}
\hline PROPERTIES & COMPOSITIONS & CRUDE & MERCERIZED & ACETYLATED \\
\hline & 10 RH/10 SOPFB & 22.50 & 29.66 & 33.83 \\
TENSILE & $10 \mathrm{RH} / 20$ SOPFB & 25.00 & 28.16 & 28.50 \\
STRENGTH (MPa) & 20RH/10 SOPFB & 24.00 & 27.10 & 28.83 \\
& $20 \mathrm{RH} / 20$ SOPFB & 27.27 & 28.66 & 28.83 \\
\hline & $10 \mathrm{RH} / 10$ SOPFB & 384.40 & 313.44 & 308.89 \\
TENSILE & $10 \mathrm{RH} / 20$ SOPFB & 259.60 & 321.71 & 260.75 \\
MODULUS (MPa) & 20RH/10 SOPFB & 375.25 & 373.15 & 324.30 \\
& 20RH/20 SOPFB & 398.11 & 308.99 & 291.80 \\
\hline
\end{tabular}




\begin{tabular}{|l|l|l|l|l|}
\hline & $10 \mathrm{RH} / 10$ SOPFB & 0.54 & 0.63 & 0.82 \\
IMPACT & $10 \mathrm{RH} / 20$ SOPFB & 0.59 & 0.72 & 0.79 \\
STRENGTH (KJ/M) & 20RH/10 SOPFB & 0.59 & 0.75 & 0.74 \\
& 20RH/20 SOPFB & 0.62 & 6.82 & 0.66 \\
\hline \multirow{3}{*}{ SOMPRESSIVE } & $10 \mathrm{RH} / 10$ SOPFB & 65.89 & 51.61 & 57.73 \\
STRENGTH(MPa) & 10RH/20 SOPFB & 72.03 & 65.11 & 54.02 \\
& 20RH/10 SOPFB & 61.67 & 60.53 & 51.05 \\
\hline HARDNESS & 20RH/20 SOPFB & 61.11 & 64.48 & 45.51 \\
VALUE (BHV) & 10RH/10 SOPFB & 70.10 & 52.91 & 136.93 \\
& 20RH/10 SOPFB & 50.27 & 57.12 & 74.91 \\
\hline
\end{tabular}

Table 2 show that impact strength of the hybrid composites range from 0.54 to $0.62 \mathrm{~kJ} / \mathrm{M}$; from 0.63 to $0.82 \mathrm{~kJ} / \mathrm{M}$ and from 0.66 to $0.82 \mathrm{~kJ} / \mathrm{M}$ respectively for crude, mercerized and acetylated fiber-based composites. Impact strength of the crude fiber composite differs highly significantly from both mercerized and Acetylated fiber composites as given in Table $3(\mathrm{P}<0.01)$. There is no significant difference between the impact strength of mercerized and acetylated fiber composites. Mercerized and Acetylated fiber composites showed higher impact strength than crude fiber composites. This result could be explained by the fact that when a crack is generated due to an impact, it propagates towards a poor interfacial region [10]. Since mercerization causes a decrease in the quantity of voids and impurities, in the fiber structure, improved impact strength could be as a result of the reduction in the number of voids.

Table 3: Physical and Mechanical Properties Multiple Comparison for Hybrid Composites with fillers from Different Chemically Modified fibers

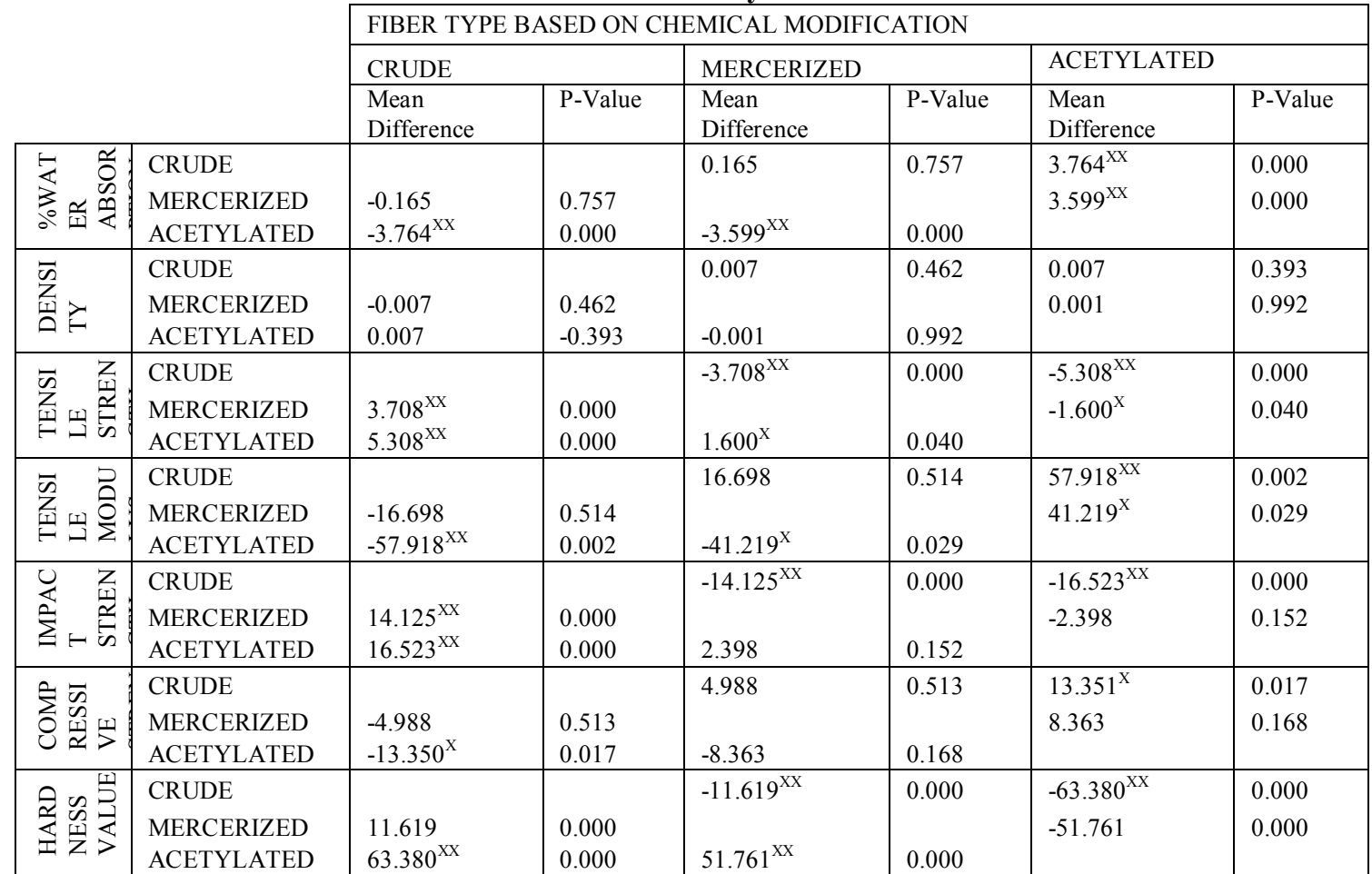

$\mathrm{X}$; the mean difference is significant at 0.05 levels $\quad \mathrm{XX}$; the mean difference is highly significant at 0.01 level

Compressive strength of hybrid composites range from 61.11 to $72.03 \mathrm{MPa}$; from 51.61 to $65.11 \mathrm{MPa}$ and from 45.51 to $57.73 \mathrm{MPa}$ respectively for crude, mercerized and acetylated fiber-based composites. Analysis of variance for compressive strength is shown in Table 3. Crude fiber-based composites differ significantly from Acetylated fiber-based composites $(\mathrm{P}<0.05)$. There is no significant difference between crude and mercerized fiber-based composites $(\mathrm{P}>0.05)$. Crude and mercerized fiber-based composites showed higher compressive strength than acetylated fiber-based composites. This could be as a result of weakening of the fiber during acetylation.

Hardness values (BHV) obtained from the tests range from 31.91 to 70.10 ; from 52.91 to 90.53 and from 74.91 to 164.30 respectively for crude, mercerized and acetylated fiber-based composites. Table 3 shows that the hardness value of the crude, mercerized and acetylated fiber-based composites differs highly significantly from each other $(\mathrm{P}<0.01)$. The mean difference shows that acetylated fiber-based composites have 
the highest hardness value followed by mercerized fiber composites. This could be as a result of similar reason advanced for same trend in tensile strength.

\section{Conclusion}

The result of this study reveals that chemical modification altered the physical and mechanical properties of "Stripped Oil Palm Fruit Bunch and Rice Husk as reinforcement in Polypropylene Hybrid composite". Acetylating reduced percentage water absorption of the fiber composite. It also, significantly, improved the tensile strength, impact strength and Hardness values while compressive strength and tensile modulus decreased. Mercerization improved the tensile strength, impact strength and hardness values of the fiber composite. Mercerization has no significant effect on the compressive strength, tensile modulus and percentage water absorption. Generally, good fiber-matrix interaction which results from chemical modification improved the properties of the fiber composite. Application of each chemically modified fiber will depend on the desired properties.

\section{Acknowledgements}

The authors wish to acknowledge the authorities of Bayero University, Kano, Obafemi Awolowo University IleIfe and Modibbo Adama University of Technology, Yola for giving them access to their equipment during the course of carrying out this work.

\section{References}

[1]. Maya, J. J., Rajesh, D. and Anandjiwala. Recent Development in Chemical Modification and Characterisation of Natural FiberReinforced Composite, Polymer Composites, 29, 2008, 187-207.

[2]. Joseph, S., Oommen, Z. and Thomas, S., Environmental Durability of Banana Fiber-Reinforced Phenol Formaldehyde Composite, Journal of Applied Polymer Science, 100, 2006, 2521-2531.

[3]. Sjorstrom, E., Wood chemistry: fundamentals and applications. (London: Academic Press, 1981).

[4]. Nevell, T. P. and Zeronian, S. H.,. Wood Fibers, (New York: New York Wiley Press1985).

[5]. Lina, H., Selvum, P. and Uday, V., Banana Fiber Composites for Automotive and Transportion Applications, SPE Conference Proceedings, Department of Materials Science and Engineering University of Alabama at Birmingham, Birminghan, AL 35294, $2009,2-10$

[6]. Maya, J. J., Rajesh, D., Anandjiwala. and Sabu T., Hybrid Composites. Journal of Bioenergy, 1, 2007, 317-329.

[7]. Namessan, N.O., Assessing the Suitability of Kenaf (Hibiscus Canabis) Fiber Material Use Industrially for the Production of Automotive Brake Pad, unpublished doctoral thesis, Department of Agricultural Engineering, Federal University of Technology, Yola., 2007.

[8]. Yang, H. S., Kim, H. J., Son, J., Park, H. J., Lee, B. J. and Hwang, T. S., Rice Husk Flour Filled Polypropylene Composite: Mechanical and Morphological Study, Composite Structure Journal, 64, 2004, 305-312.

[9]. Edeerozey, A.M., Akil, H.M., Azhar, A.B. and Ariffin, M.Z., Chemical Modification of Kenaf Fibers, Materials Letters, 61, 2007, 2023-2025.

[10]. Hattotuwa, G.B.P., Ismail, H. and Baharin, A., Comparison of the Mechanical Properties of Rice Husk Powder Filled Polypropylene Composites with Talc Filled Polypropylene Composites, Polymer Testing, 21, 2002, 833-839. 